\title{
Challenges in diagnosing pediatric malaria in Dar es Salaam, Tanzania
}

\author{
Gro EA Strom ${ }^{1 *}$, Christel G Haanshuus ${ }^{3}$, Sabrina Moyo ${ }^{1,2}$, Maulidi Fataki ${ }^{2}$, Nina Langeland ${ }^{1}$, Bjørn Blomberg ${ }^{1,3}$ \\ From Challenges in malaria research \\ Basel, Switzerland. 10-12 October 2012
}

\section{Background}

Malaria is a major cause of pediatric morbidity and mortality. No clinical features clearly differentiate malaria from other causes of pediatric febrile illness. Lack of laboratory equipment and expertise makes malaria diagnostics a challenge, leading to overdiagnosis and overtreatment of malaria. Molecular methods (PCR) are emerging but have so far not proven practical for routine clinical use. Emerging antimalarial and antibiotic resistance calls for precise diagnostics and treatment of febrile illness, and has led WHO to recommend laboratory confirmation of malaria in children before starting treatment.

\section{Methods}

Children admitted with fever were recruited consecutively among admissions at the general pediatric wards at Muhimbili National Hospital (MNH) in Dar es Salaam Tanzania from January-June 2009. Clinical, demographic and laboratory features were registered. Microscopy of thick blood smears was done as part of the routine at $\mathrm{MNH}$, and thin blood smears were stained and examined later. Retrospectively, genus-specific PCR of Plasmodium mitochondrial DNA was performed on DNA extracted from whole blood for all patients and species-specific PCR was done on samples positive by genus-specific PCR. Univariate and multivariate statistical analysis was performed using IBM SPSS Statistics version 19 (SPSS Inc, IBM Company).

\section{Results}

The study included 304 children. Within four weeks before admission $62.6 \%$ had received antimalarials. Forty children had positive routine thick blood smears upon admission and twenty had positive research thin blood smears upon retrospective examination. Twenty-five

${ }^{1}$ Institute of Medicine, University of Bergen, Bergen, Norway

Full list of author information is available at the end of the article percent had positive PCR, all positive for $P$. falciparum. PCR results confirmed positive routine microscopy in only $52,5 \%$ and research microscopy in $100 \%$. Almost every fifth febrile child (55/304) had positive PCR but negative research microscopy. High parasitemia on routine microscopy was associated with positive research microscopy and positive PCR. The true prevalence of malaria in the population remains unknown as none of the diagnostic methods can be interpreted as a true gold standard. Palmar pallor, low hemoglobin and low platelet count were significantly associated with both positive PCR and positive research microscopy $(\mathrm{p}<0.001)$. In hospital, $65.1 \%$ received antimalarial treatment. Clinically determined severity of palmar pallor was clearly associated with hemoglobin level.

\section{Conclusions}

The study identified discrepancies between routine malaria microscopy, research malaria microscopy and PCR. Almost half of routine microscopy positive cases were negative on PCR, indicating prevalent overdiagnosis of malaria. PCR was positive for many research microscopy negative cases. This may in part be due to prevalent treatment with antimalarials before admission. Palmar pallor and low hemoglobin levels were predictors for malaria in this study. The current routine diagnostic method for malaria appears to lead to overdiagnosis of malaria and, consequently, overuse of antimalarials. Conversely, children with false positive malaria diagnosis may die because they do not receive treatment for the true cause of their illness. Malaria is still a prominent health issue in Tanzania, and the uncertainty of both clinical and routine laboratory tests may lead to misuse of antimalarials and antibiotics and consequently contribute to emerging drug resistance. Diagnostic algorithms employing new methods such as rapid diagnostic tests (RDTs) may contribute to improving malaria diagnosis and treatment. 


\section{Author details}

'Institute of Medicine, University of Bergen, Bergen, Norway.

${ }^{2}$ Muhimbili University of Health and Allied Sciences, Dar es Salaam, Tanzania.

${ }^{3}$ Department of Medicine, Haukeland University Hospital, Bergen, Norway.

Published: 15 October 2012

doi:10.1186/1475-2875-11-S1-P92

Cite this article as: Strom et al:: Challenges in diagnosing pediatric

malaria in Dar es Salaam, Tanzania. Malaria Journal 2012 11(Suppl 1):P92.

Submit your next manuscript to BioMed Central and take full advantage of:

- Convenient online submission

- Thorough peer review

- No space constraints or color figure charges

- Immediate publication on acceptance

- Inclusion in PubMed, CAS, Scopus and Google Scholar

- Research which is freely available for redistribution

Submit your manuscript at www.biomedcentral.com/submit 